\title{
THE INFLUENCE OF THE KOSTERLITZ-THOULESS TRANSITION ON THE DECAY OF SPIN-POLARIZED ATOMIC HYDROGEN
}

\author{
H.T.C. Stoof and M. Bijlsma \\ Department of Theoretical Physics, Eindhoven University of Technology, \\ P.O. Box 513, 5600 MB Eindhoven, The Netherlands
}

Using our microscopic theory of the two-dimensional dilute Bose gas, we confirm the conjecture by Kagan $e t$ al. that the rate constant for three-body recombination experiences a discontinuity at the critical temperature of the Kosterlitz-Thouless transition and calculate the reduction of the recombination rate as a function of temperature and density. Besides the jump in the adsorbtion isotherm, this effect is also an unambiguous signal for the achievement of the Kosterlitz-Thouless transition in spin-polarized atomic hydrogen adsorbed on a superfluid helium film.

\section{INTRODUCTION}

It has been recognized for some time that doubly spin-polarized atomic hydrogen is a very suitable candidate for the observation of macroscopic quantum phenomena in a weakly interacting Bose gas. Up to now all experimental effort has been focussed on the achievement of Bose-Einstein condensation, but recently also the possibility of attaining the Kosterlitz-Thouless transition in atomic hydrogen gas adsorbed on a superfluid helium film was discussed [1].

In the two-dimensional case there are at least two phenomena that are of importance for the actual observation of the phase transition. One is the jump in the adsorbtion isotherm predicted by Svistunov et al. [1] and the other is the decrease of the three-body dipolar recombination rate. With respect to the latter, Kagan et al. [2] found (but see below) that the value of the rate constant near zero temperature is a factor of six smaller than just above the critical temperature of the Kosterlitz-Thouless transition. Moreover, they speculated on the possibility of a discontinuous behavior at the critical point but their discussion is based on the Popov theory of the dilute Bose gas $[3,4]$ and is therefore confined to temperatures far below the critical one.

However, the issue of the discontinuity together with the estimate of the magnitude of the effect can be conveniently discussed within the framework of the T-matrix or ladder approximation, which gives an accurate description of the dilute gas at essentially all temperatures [4]. This is the purpose of the present paper.

\section{RECOMBINATION}

Using a Jastrow-like approximation [5] for the initial-state wavefunction in the recombination matrix element we find for the rate constant in the normal phase

$\mathrm{L}(\mathrm{T})=\mathrm{L}_{0}\left|\frac{\mathrm{m}}{\hbar^{2}} \mathrm{~T}^{2 \mathrm{~B}}(0,0 ;-2 \hbar \Sigma)\right|^{6}$,

with $\mathrm{T}^{2 \mathrm{~B}}(0,0 ; \mathrm{E})$ the two-body $\mathrm{T}$ matrix at energy $E$ and $m$ the mass of the hydrogen atom. Furthermore, the selfenergy $\hbar \Sigma$ is determined from [4]

$\hbar \Sigma=2 n T^{2 \mathrm{~B}}(0,0 ;-\hbar \Sigma)$

and thus only depends on the density $n$ of the gas. Note that to arrive at these expressions we have neglected the contribution of the kinetic energy to the energy argument of the $T$ matrix, which restricts the use of this result to just above the critical temperature.

In the superfluid phase two modifications to the decay rate are necessary. First, the right-hand side of Eq. (1) is multiplied by the renormalized correlator [2]

$K_{R}(T)=\frac{1}{6 n^{3}}\left\{6 n^{3}-9 n_{0}^{2}+4 n_{0}^{3}\right\}$ 
and second, the two-body $\mathrm{T}$ matrix is replaced by the many-body $T$ matrix since the collision of two atoms in the gas is affected by the presence of the quasicondensate density $n_{0}[4]$. Hence,

$L(T)=L_{0} \mid \frac{m}{\hbar^{2}} T_{(0,0,0 ; 0)}^{M B} K_{R}(T)$

below the Kosterlitz-Thouless transition.

Clearly, a similar expression can be given for the two-body dipolar relaxation rate constant. However, we restrict ourselves in the following to a discussion of the recombination process, which is the dominant decay mechanism at the high densities required for the observation of the superfluid phase.

\section{RESULTS AND DISCUSSION}

The results of the above approach are summarized in Fig. 1. First of all we calculate the ratio of the recombination rate in the normal and superfluid phase as a function of temperature at a fixed two-dimensional density. At low temperatures the many-body $\mathrm{T}$ matrix is nearly constant and this ratio slightly decreases with temperature, because the correlator increases if the quasicondensate density diminishes. At higher temperatures the ratio sharply rises due to the enhanced influence of the quasiparticles, which reduces the many-body $T$ matrix by almost a factor of three at the critical temperature. Notice that at zero temperature the reduction of the rate constant is more than a factor of six, since in this limit the many-body $T$ matrix equals the two-body $T$ matrix at energy $-\hbar \Sigma$, which is smaller than at $-2 \hbar \Sigma$. This effect, which also follows from the Popov theory, leads to an additional reduction by a factor of two at the density considered here.

From an experimental point of view it is interesting to consider the situation of a two-dimensional gas in thermal contact with a buffer volume [1]. Therefore, we also calculate the discontinuity in the recombination rate constant as a function of the critical temperature in these circumstances, by taking the jump in the adsorbtion isotherm into account. We then find a

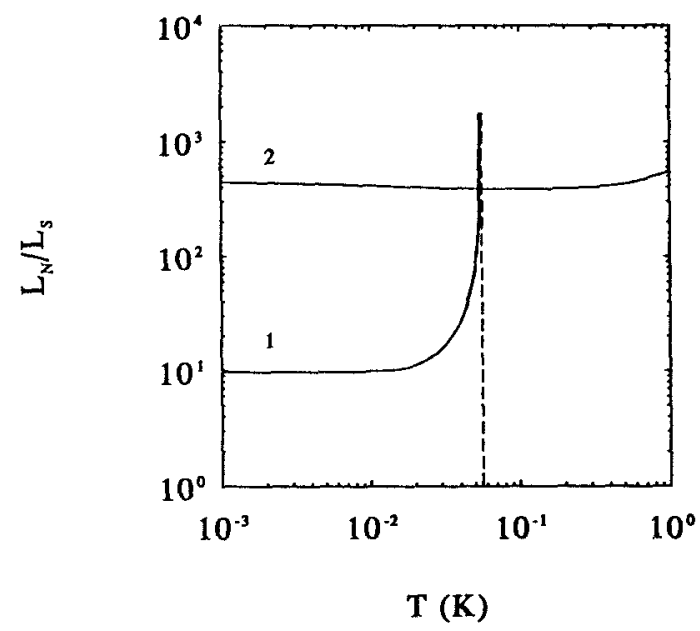

Figure 1. Ratio of the decay rate constant in the normal and superfluid phase (1) at a fixed density of $10^{13} \mathrm{~cm}^{-2}$ and (2) at the critical point of a gas in thermal contact with a buffer volume.

reduction by a factor of about 400 . However, this might be an overestimate of the effect, since fluctuations beyond the ladder approximation can be of importance in the critical region [4]. Nevertheless, the reduction is certainly substantially larger than anticipated from the correlator alone and should be clearly visible in future experiments with atomic hydrogen.

We thank Meritt Reynolds and Jook Walraven for a stimulating discussion leading to this work.

\section{REFERENCES}

1. B.V. Svistunov, T.W. Hijmans, G.V. Shylapnikov, and J.T.M. Walraven, Phys. Rev. B43, 13412 (1991).

2. Yu. Kagan, B.V. Svistunov, and G.V. Shylapnikov, Sov. Phys. JETP 66, 314 (1987).

3. V.N. Popov, Functional Integrals in Quantum Field Theory and Statistical Physics (Reidel, Dordrecht, 1983), Chap. 6.

4. H.T.C. Stoof and M. Bijlsma, Phys. Rev. E47, 939 (1993).

5. H.T.C. Stoof, L.P.H. de Goey, W.M.H.M. Rovers, P.S.M. Kop Jansen, and B.J. Verhaar, Phys. Rev. A38, 1248 (1988). 